\title{
Analysis of the Coupling Coefficient in Inductive Energy Transfer Systems
}

\author{
Rafael Mendes Duarte and Gordana Klaric Felic \\ The University of Melbourne, Parkville, VIC 3010, Australia \\ Correspondence should be addressed to Rafael Mendes Duarte; rmendesduarte@gmail.com
}

Received 8 January 2014; Accepted 4 June 2014; Published 17 June 2014

Academic Editor: Gerard Ghibaudo

Copyright ( 2014 R. Mendes Duarte and G. Klaric Felic. This is an open access article distributed under the Creative Commons Attribution License, which permits unrestricted use, distribution, and reproduction in any medium, provided the original work is properly cited.

\begin{abstract}
In wireless energy transfer systems, the energy is transferred from a power source to an electrical load without the need of physical connections. In this scope, inductive links have been widely studied as a way of implementing these systems. Although high efficiency can be achieved when the system is operating in a static state, it can drastically decrease if changes in the relative position and in the coupling coefficient between the coils occur. In this paper, we analyze the coupling coefficient as a function of the distance between two planar and coaxial coils in wireless energy transfer systems. A simple equation is derived from Neumann's equation for mutual inductance, which is then used to calculate the coupling coefficient. The coupling coefficient is computed using CST Microwave Studio and compared to calculation and experimental results for two coils with an excitation signal of up to $10 \mathrm{MHz}$. The results showed that the equation presents good accuracy for geometric parameters that do not lead the solution of the elliptic integral of the first kind to infinity.
\end{abstract}

\section{Introduction}

Wireless power systems transfer the energy without wire connection between a source and a load. This provides several advantages specially for applications involving implantable devices $[1,2]$ or devices that need to be hermetically sealed during operation [3]. For the first, the use of batteries presents issues in terms of periodic replacement which may require complex surgical procedures. For the latter, a connection through wires becomes unfeasible. Among the many classes of wireless systems, energy transfer via inductive link has been widely studied due to high efficiency and high transmission power capability [4].

In inductive coupled systems, a power amplifier supplies an alternative current to the primary coil, which produces a magnetic field at the secondary coil. The produced field induces a current and voltage at the secondary branch, which can be rectified and supplied to a load. An important parameter for inductive links is the coupling coefficient $k$, which measures how much power from the generated electromagnetic (EM) field is induced in the secondary coil. The coupling coefficient is related to the reflected impedance from the secondary on the primary side. Therefore, variations on $k$ change the output impedance of the power amplifier, removing it from its optimum operation condition and decreasing its efficiency [5].

Previous studies on the magnetic coupling suggest that the coupling coefficient can be affected mostly due to variations in the distance between the coils [6], relative angle between the coils, and magnetic properties of the material surrounding the coils. This is particularly important for systems where the two coils are not in a fixed relative position. For such systems, the efficiency can be seriously compromised due to inefficient operation of the power amplifier. Hence, analyzing the behavior of the coupling factor as a function of these critical parameters can help to maintain the system efficiency stable as the coupling between the coils varies.

Numerical simulations provide highly accurate results but require long computational time and extensive memory usage. Although the analytical methods based on the closed form expressions lack high accuracy, they offer fast solutions and explicit control over the design parameters. 


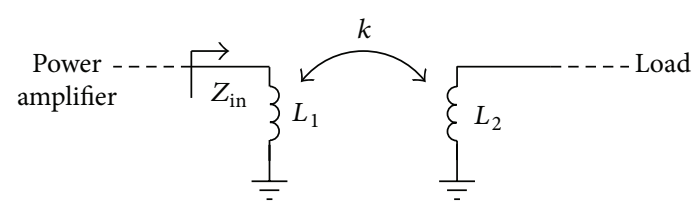

FIgURE 1: Inductive link schematic.

In this paper, we derive a simple formula for the mutual inductance as a function of the distance between two planar and coaxial coils. The equation is obtained from Neumann's equation for the mutual inductance, using power series as approximated solutions of the elliptic integrals, which are usually computationally expensive to obtain. We also give theoretical insight about the correlation between magnetic field distribution and coupling coefficient. The presented equation is compared to simulation and experimental results of two designed coils with excitation signals at frequencies of up to $10 \mathrm{MHz}$.

\section{Inductive Link Systems}

In Figure 1, a simple diagram of an inductive link is shown. A primary coil is excited by a power amplifier (PA), usually operating in class $\mathrm{E}$ due to its high efficiency. Power is transmitted to the load via magnetic coupling between inductors $L_{1}$ and $L_{2}$. The system efficiency is defined as the ratio between the power dissipated on the load and the power supplied to the power amplifier. In addition to losses in the inductors, due to finite resistance of the coils, the PA efficiency plays a major role in degrading overall efficiency.

In order to design the PA, the impedance seen at its output $\left(Z_{\text {in }}\right.$ in Figure 1$)$ must be taken into account. In most designs, the inductance of the primary coil is used as a component for the output filter of the power amplifier while the output resistance is considered the load of the amplifier. The values of these output components are tightly related to the amplifier efficiency, once optimum operation state is achieved for determined component values [5]. Therefore, variations on the output impedance can significantly decrease the amplifier efficiency and, as a consequence, the system efficiency.

For a generic load $Z_{L}$ connected to the secondary coil, the impedance $Z_{\text {in }}$ can be defined as

$$
Z_{\text {in }}=R_{1 S}+j w L_{1}+\frac{w^{2} M^{2}}{j w L_{2} R_{2 S}+Z_{L}},
$$

where $R_{1 S}$ represents the series resistance of $L_{1}$ and $M$ is mutual inductance, related to the coupling coefficient $k$ according to

$$
k=\frac{M}{\sqrt{L_{1} L_{2}}},
$$

where $L_{1}$ and $L_{2}$ are the self-inductance of coils 1 and 2 .

Equations (1) and (2) express the dependence of the output impedance on the coupling coefficient of the inductive link. As the mutual inductance varies, the third term of

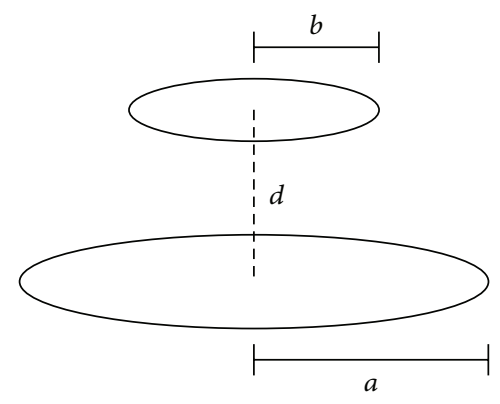

FIgURE 2: Two coaxial coils with radii $a$ and $b$.

(1), which can have an imaginary and real part, varies and modifies the impedance $Z_{\text {in }}$ shown in Figure 1.

\section{Coupling Coefficient Calculation}

The mutual inductance between two circular loops separated by a distance $d$ and with radii $a$ and $b$, as depicted in Figure 2, can be calculated using Neumann's equation [7]:

$$
M=\frac{\mu}{4 \pi} \iint \frac{\cos \epsilon}{r} d s d s^{\prime},
$$

where $d s$ and $d s^{\prime}$ are the incremental sections of the circular filaments and $r$ is the distance between these two sections, which are defined as

$$
\begin{gathered}
r=\sqrt{a^{2}+b^{2}+d^{2}-2 a \cos \left(\phi-\phi^{\prime}\right)}, \\
\epsilon=\phi-\phi^{\prime}, \quad d s=a d \phi, \quad d s=b d \phi^{\prime} .
\end{gathered}
$$

The substitution of (4) in (3) results in

$$
M=\frac{\mu}{4 \pi} \iint_{0}^{2 \pi} \frac{a b \cos \left(\phi-\phi^{\prime}\right)}{a^{2}+b^{2}+d^{2}-2 a \cos \left(\phi-\phi^{\prime}\right)} d \phi d \phi^{\prime} .
$$

The integral in (5) can be rewritten using elliptic integrals, yielding

$$
M(m)=\frac{2 \mu \sqrt{a b}}{m}\left[\left(1-\frac{m^{2}}{2}\right) K(m)-E(m)\right],
$$

where $K(m)$ and $E(m)$ are the elliptic integrals of first and second kind, respectively, and $m$ is defined as

$$
m=\sqrt{\frac{4 a b}{(a+b)^{2}+d^{2}}}
$$

assuming values between 0 and 1 .

3.1. Solution of the Elliptic Integrals. The solutions of the elliptic integrals of the first and second kind can be approximated [8] using (8) and (9). The approximation and the solution obtained through numeric integration are depicted in Figures 3 and 4 . For low values of $m$, the power series representation shows reasonable accuracy. However, $m$ increases both curves 


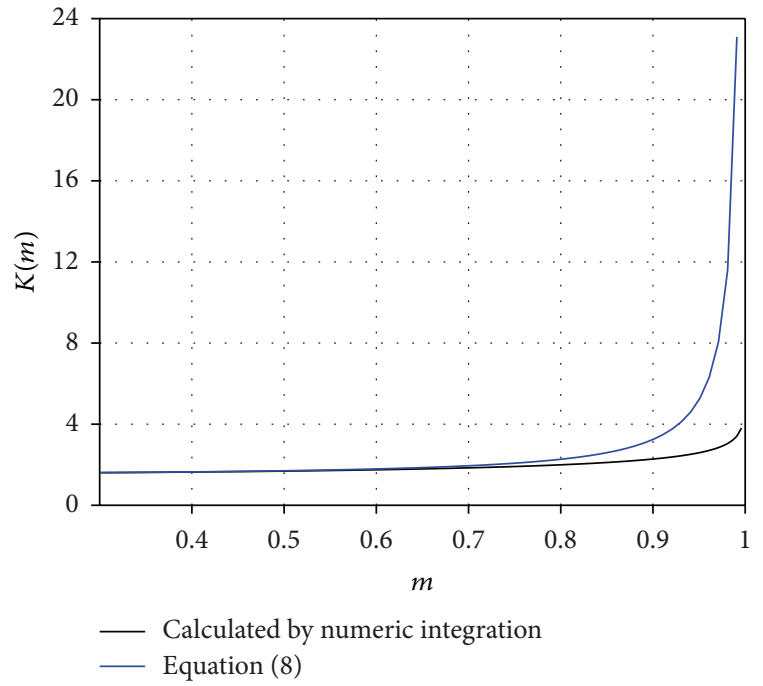

FIGURE 3: Comparison between numerical integration and power series approximation for elliptic integral of the first kind.

diverge from the numeric integration values. For the elliptic integral of the first kind, as $m$ approaches the unity, the solution asymptotically tends to infinity much faster than the solution calculated by numeric integration:

$$
\begin{gathered}
K(m)=\frac{\pi}{2}+\frac{\pi}{8} \frac{m^{2}}{1-m^{2}}, \\
E(m)=\frac{\pi}{2}-\frac{\pi}{8} m^{2} .
\end{gathered}
$$

The second term of (8) tends to infinity as $m$ tends to 1 :

$$
m=\sqrt{\frac{4 a b}{(a+b)^{2}+d^{2}}}=1
$$

which leads to the following relation between the coils radius and the distance between the coils:

$$
d^{2}=(a-b)^{2} .
$$

Hence, (8) is not valid when the distance between the two coils approaches the value expressed by (11). In order to avoid this limitation, a high number of terms would be required for the series approximation (8), making the final expression of the mutual inductance too complex and extensive. Alternatively, the solution of the elliptic integral of the first kind can be approximated by a logarithm function [8]. However, the logarithm approximation leads to issues related to the signal of (6) and to an extensive resultant expression for the mutual inductance. Therefore, in this paper, we only use approximations (8) and (9), analyzing its limitations.

3.2. Mutual Inductance Calculation. The substitution of (8) and (9) in (6) yields

$$
M(m)=\frac{\mu \pi \sqrt{a b}}{8} \frac{m^{3}}{1-m^{2}} .
$$

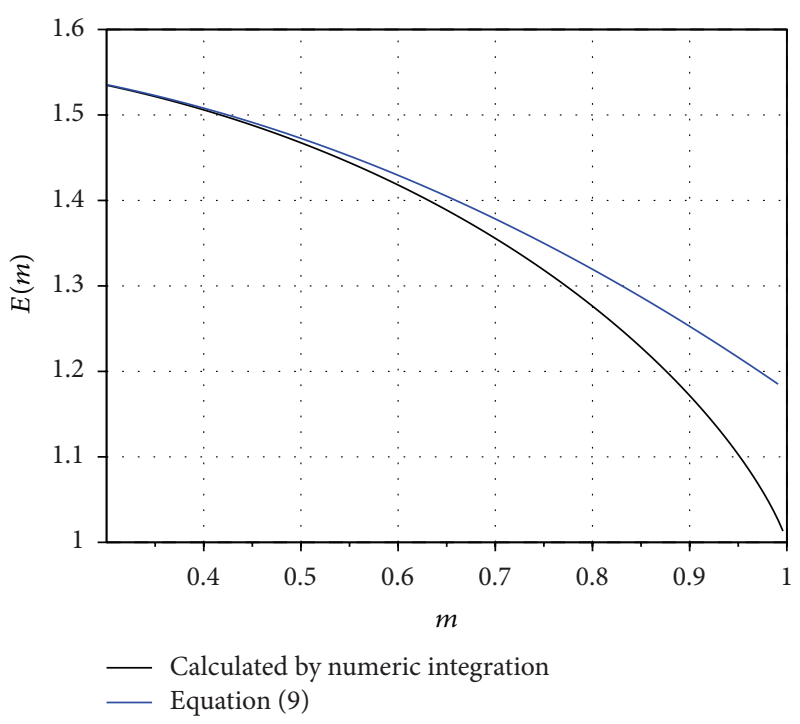

FIgURE 4: Comparison between numerical integration and power series approximation for elliptic integral of the second kind.

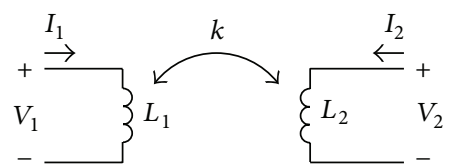

FIGURE 5: Circuit schematic of the inductive link.

Next, substituting (7) in the expression above results in the expression for the mutual inductance as a function of the distance between two circular coaxial loops:

$$
M=\frac{\mu \pi a^{2} b^{2}}{\sqrt{(a+b)^{2}+d^{2}}\left[(a-b)^{2}+d^{2}\right]} .
$$

For two coils with $n_{1,2}$ turns, the expression can be adjusted [6], yielding

$$
M=\frac{\mu \pi n_{1} n_{2} a^{2} b^{2}}{\sqrt{(a+b)^{2}+d^{2}}\left[(a-b)^{2}+d^{2}\right]}
$$

which express the mutual inductance of two coils with $n_{1,2}$ as a function of distance $d$, the magnetic permeability of the material surrounding the coils $\mu$ and the inner radius of the two coils.

\section{Coupling Coefficient Measurement Technique}

In this section, we discuss the methodology used to infer the coupling coefficient in simulations. The circuit schematic of the inductive link is shown in Figure 5 and its equations can be written as

$$
\begin{aligned}
& V_{1}=j w\left(I_{1} L_{1}+M I_{2}\right), \\
& V_{1}=j w\left(I_{2} L_{2}+M I_{1}\right) .
\end{aligned}
$$


Assuming the secondary coil is shorted, (16) becomes

$$
I_{2}=-\frac{M I_{1}}{L_{2}}
$$

Substituting (17) in (15) yields

$$
V_{1}=j w I_{1}\left(L_{1}-\frac{M}{L_{2}}\right)
$$

Assuming

$$
L_{s}=L_{1}-\frac{M}{L_{2}}
$$

equation (18) becomes

$$
V_{1}=j w I_{1} L_{s}
$$

Hence, the inductance $L_{s}$ is the inductance measured when the secondary coil is shorted. Rearrange (19) in terms of the mutual inductance:

$$
M=L_{2}\left(L_{1}-L_{s}\right)
$$

Finally, combining (21) and (2) results in the expression of the coupling coefficient in terms of the self-inductance $L_{1}$ and the inductance measured when the secondary coil is shorted, $L_{s}$ :

$$
k=\sqrt{1-\frac{L_{s}}{L_{1}}}
$$

\section{Results}

Simulation results were used to check the accuracy of (14). In addition, experimental results were analyzed for two coils with geometries that lead the coefficient $m$ close to unity for short separation distances between the coils.

5.1. Mesh Properties. To evaluate the coil impedance, it is necessary to take into account the geometry and curvature of the coils, as well as the interaction between the traces of each turn. Thus, circular coils are computational expensive since a very fine mesh is required to precisely create the EM model for the circular shapes of the coils. In order to achieve reasonable accuracy and reliable results, the mesh cell size was decreased to a point where no further variation in the results was observed. In addition, the accuracy of curvature elements in CST was also increased until stable results were achieved.

5.2. Simulation. In order to analyze the accuracy of (14), the coupling coefficient between two coils, with dimensions given by Table 1 , was computed using CST. Parameters $s$ and $w$ are the spacing between traces and trace width, respectively; $h$ is the trace height and $n$ is the number of turns. The coupling coefficient was measured according to the method explained in Section 4, for an excitation signal at 1, 6, and $10 \mathrm{MHz}$. The results are shown in Figure 6.
TABLE 1: Dimensions of simulated coils (in mm).

\begin{tabular}{lccccc}
\hline & $a$ & $h$ & $w$ & $s$ & $n$ \\
\hline Coil 1 & 11.5 & 0.035 & 0.5 & 0.5 & 7 \\
Coil 2 & 11.5 & 0.035 & 0.5 & 0.5 & 4 \\
\hline
\end{tabular}

TABLE 2: Dimensions of coils used for measurements (in mm).

\begin{tabular}{lcccc}
\hline$a$ & $h$ & $w$ & $s$ & $n$ \\
\hline 23.5 & 0.035 & 0.5 & 0.5 & 18 \\
\hline
\end{tabular}

Equation (14) presented a reasonable accuracy compared to simulation results, specially for large separation distance between the coils ( $d>20 \mathrm{~mm}$ ). Also, as (14) predicts, the coupling coefficient is not dependent on the operation frequency, presenting small variations at different frequencies. These small variations can be explained by proximity and skin effects, which are responsible for slightly changing the impedance of the primary and secondary coils. The skin effect increases proportionally to the square root of the operation frequency, increasing the impedance of the coil.

5.3. EM Field Distribution. Figure 7 shows the magnetic field distribution surrounding the coils at $6 \mathrm{MHz}$. The field lines are normal to the $X Y$ plane. The coupling coefficient is dependent on how much of the magnetic field flux is encircled by the secondary coil, being higher if a high density of lines is projected through the secondary coil. The amount of magnetic field lines diverging from the secondary coil can be seen as a field leakage, reducing the coupling between the two coils.

As the two coils are separated apart in the $Z$ direction, more field leakage is observed as the concentration of field lines reaching the secondary coil becomes lower.

5.4. Measurements. The limitation of (14) was also tested through experiments. We measured the coupling coefficient of two identical coils with dimensions given in Table 2. The setup for the measurement is shown in Figure 8. The primary coil was connected to a port of a vector network analyzer and the $S$ parameters were measured. The $Z$ matrix was calculated, yielding the input impedance and, therefore, the inductance seen at the primary coil when the secondary coil is shorted $\left(L_{s}\right.$ in (22)).

The measured and calculated results are depicted in Figure 9. The measurements were performed at an operation frequency of $1 \mathrm{MHz}$. For distances above $10 \mathrm{~mm}$, the calculated results are reasonable close to the experimental ones. Since the radii of both coils are the same, (11) can be written as

$$
d^{2}=0
$$

which means that for distances close to $0,(14)$ is no longer valid and good accuracy is no longer obtained, as shown in Figure 9 for small distances $(d<10 \mathrm{~mm})$.

As previously mentioned, as the distance becomes closer to the condition given by (23), the second term of 


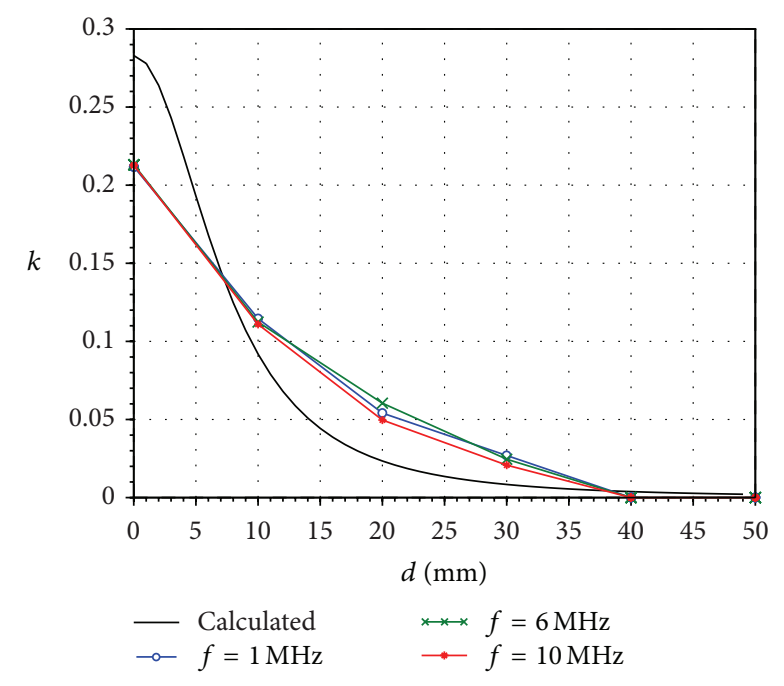

FIGURE 6: Coupling coefficient as a function of distance for different frequencies.

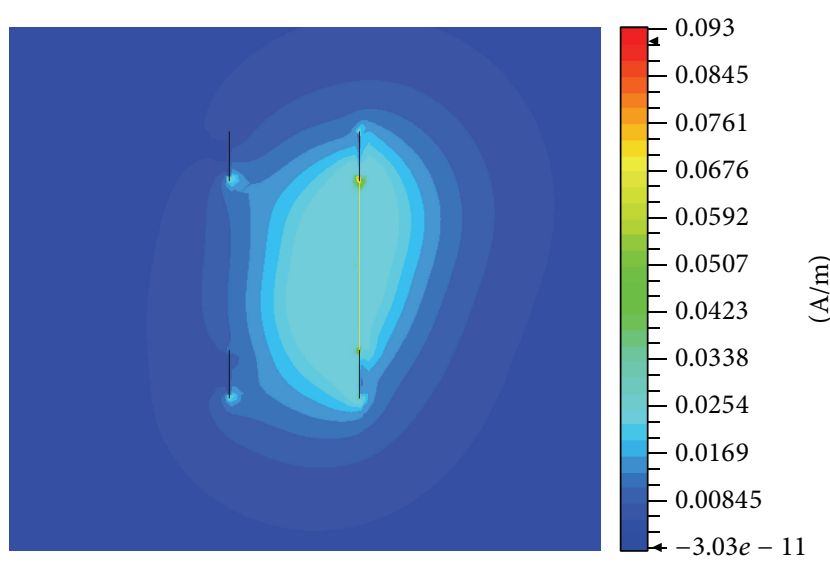

FIGURE 7: Magnetic field distribution surrounding the coils, in $Y Z$ plane.

the approximation of the elliptic integral of the first kind (8) asymptotically tends to infinity, resulting in high values of the coupling coefficient.

\section{Conclusions}

In this paper, the coupling coefficient between two coplanar and coaxial coils was analyzed as a function of the separation distance. We derived an equation for the mutual inductance from Neumann's equation which can be used to estimate the coupling coefficient between the coils. In order to derive the equation, we used a power series approximation for the solution of the elliptic integrals, which is usually calculated by numerical integration. The derived equation presented good accuracy when compared to simulation results. The experimental results demonstrated the limitation of the presented equation, as it can only be used for coil geometries and properties that do not lead the solution of the elliptic integral of the first kind to infinity.

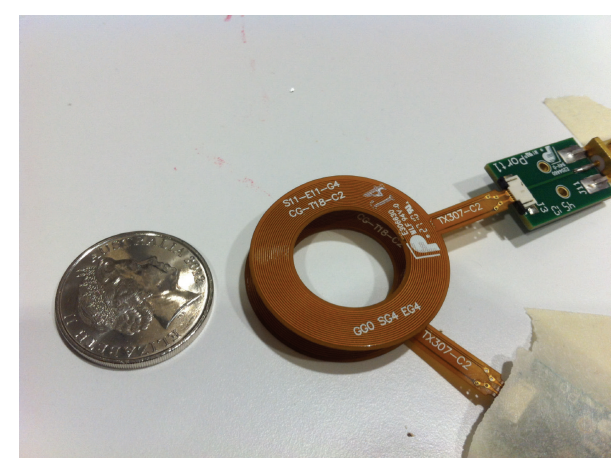

FIGURE 8: Setup for measurement of coupling coefficient.

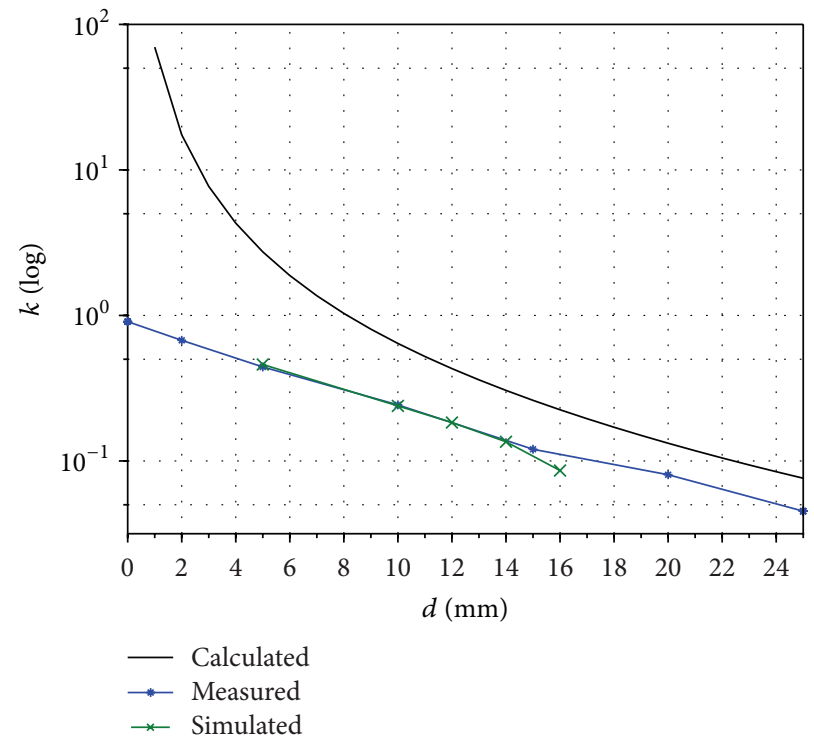

FIGURE 9: Measured coupling coefficient at $1 \mathrm{MHz}$.

The analysis presented here is useful for inductive link systems where the distance between the coils is not constant. By predicting the behavior of the coupling coefficient as a function of the variable distance, it is possible to devise a simple mechanism capable of locking the system efficiency at a desired level despite variations in the relative position of the source and load.

\section{Conflict of Interests}

The authors declare that there is no conflict of interests regarding the publication of this paper.

\section{Acknowledgments}

The authors would like to thank the Brazilian National Council for Scientific and Technological Development (CNPq) for funding the studies of the first author in Australia. Also, they would like to thank NICTA, Australia's Information and Communications Technology (ICT) Research Centre of Excellence. 


\section{References}

[1] P. Li and R. Bashirullah, "A wireless power interface for rechargeable battery operated medical implants," IEEE Transactions on Circuits and Systems II: Express Briefs, vol. 54, no. 10, pp. 912-916, 2007.

[2] Q. Ma, M. R. Haider, S. Yuan, and S. K. Islam, "Power-oscillator based high efficiency inductive power-link for transcutaneous power transmission," in Proceedings of the 53rd IEEE International Midwest Symposium on Circuits and Systems (MWSCAS '10), pp. 537-540, August 2010.

[3] I. Muller, E. P. Freitas, A. A. Susin, and C. Pereira, "Namimote: a low cost sensor node for wireless sensor network," in Internet of Things, Smart Spaces, and Next Generation Networks, S. Andreev, S. Balandin, and Y. Koucheryavy, Eds., vol. 1, pp. 391400, Springer, Berlin, Germany, 1st edition, 2012.

[4] A. Kurs, A. Karalis, R. Moffatt, J. D. Joannopoulos, P. Fisher, and M. Soljačić, "Wireless power transfer via strongly coupled magnetic resonances," Science, vol. 317, no. 5834, pp. 83-86, 2007.

[5] R. L. O. Pinto, R. M. Duarte, F. R. Sousa, I. Muller, and V. J. Brusamarello, "Efficiency modeling of class-E power oscillators for wireless energy transfer," in Proceedings of the IEEE International Instrumentation and Measurement Technology Conference (I2MTC '13), pp. 271-275, May 2013.

[6] S. F. Pichorim, "Design of circular solenoid coils for maximum mutual inductance," in Proceedings of the14th International Symposium on Biotelemetry, pp. 71-77, March 1998.

[7] J. C. Maxwell, A Treatise on Electricity and Magnetism, vol. 2, Dover, New York, NY, USA, 1954.

[8] A. Russell, "The magnetic field and inductance coefficients of circular, cylindrical, and helical currents," Proceedings of the Physical Society of London, vol. 20, no. 1, article 334, pp. 476506, 1906. 

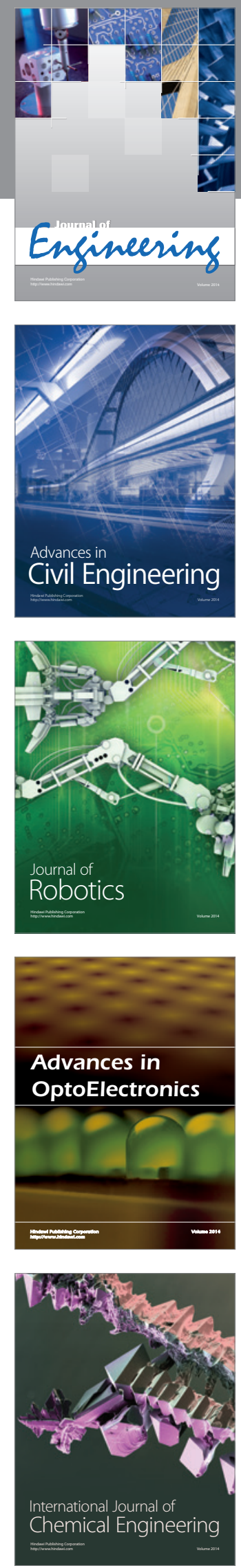

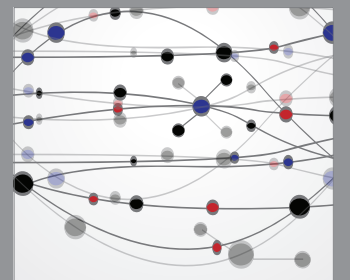

The Scientific World Journal
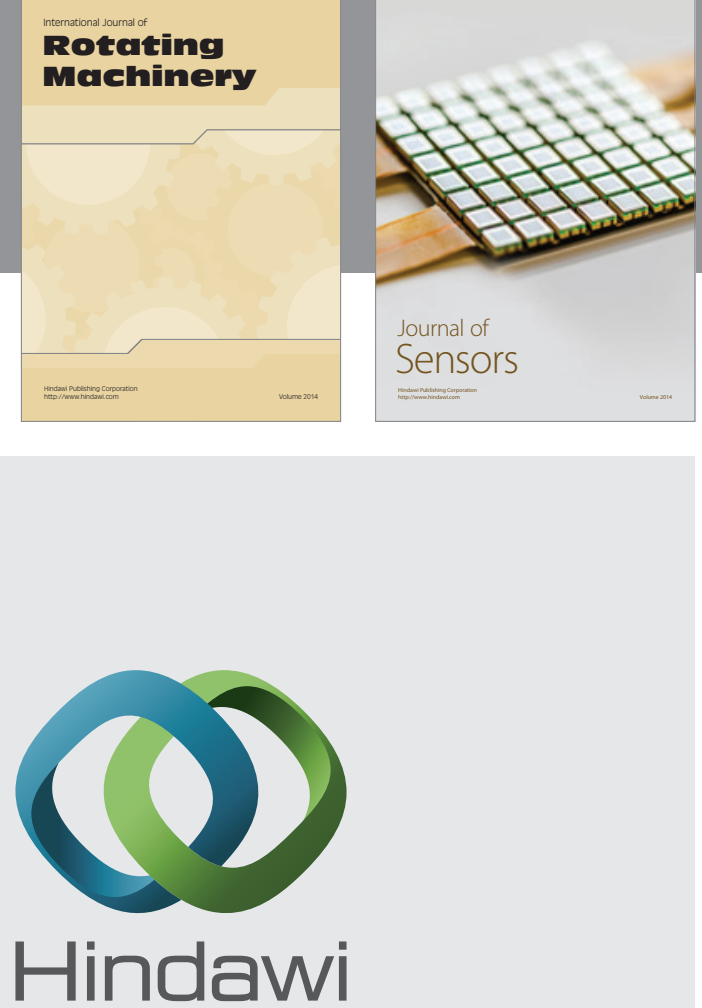

Submit your manuscripts at http://www.hindawi.com
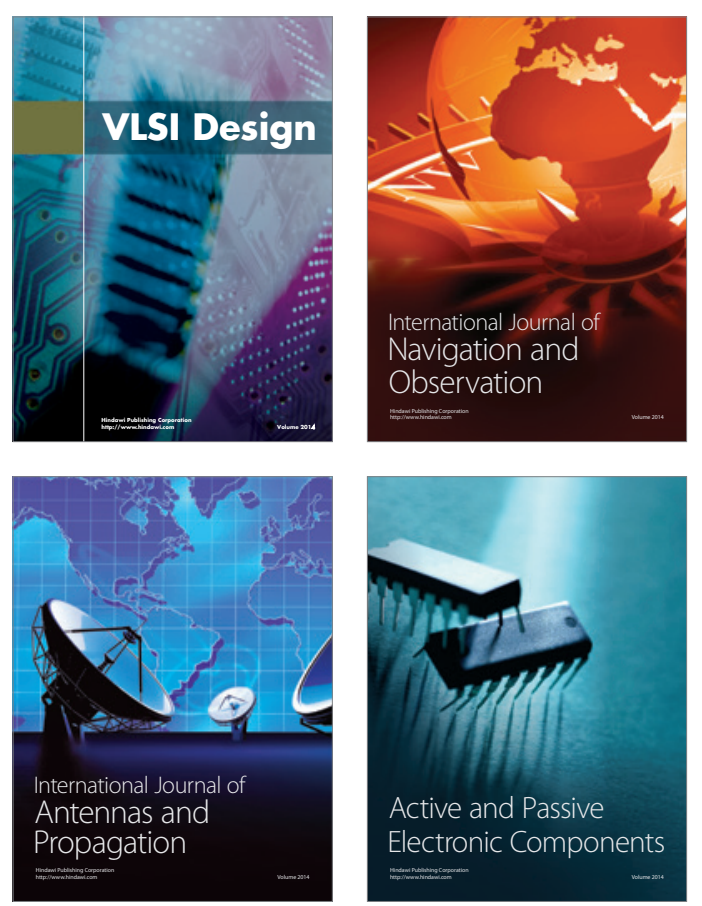
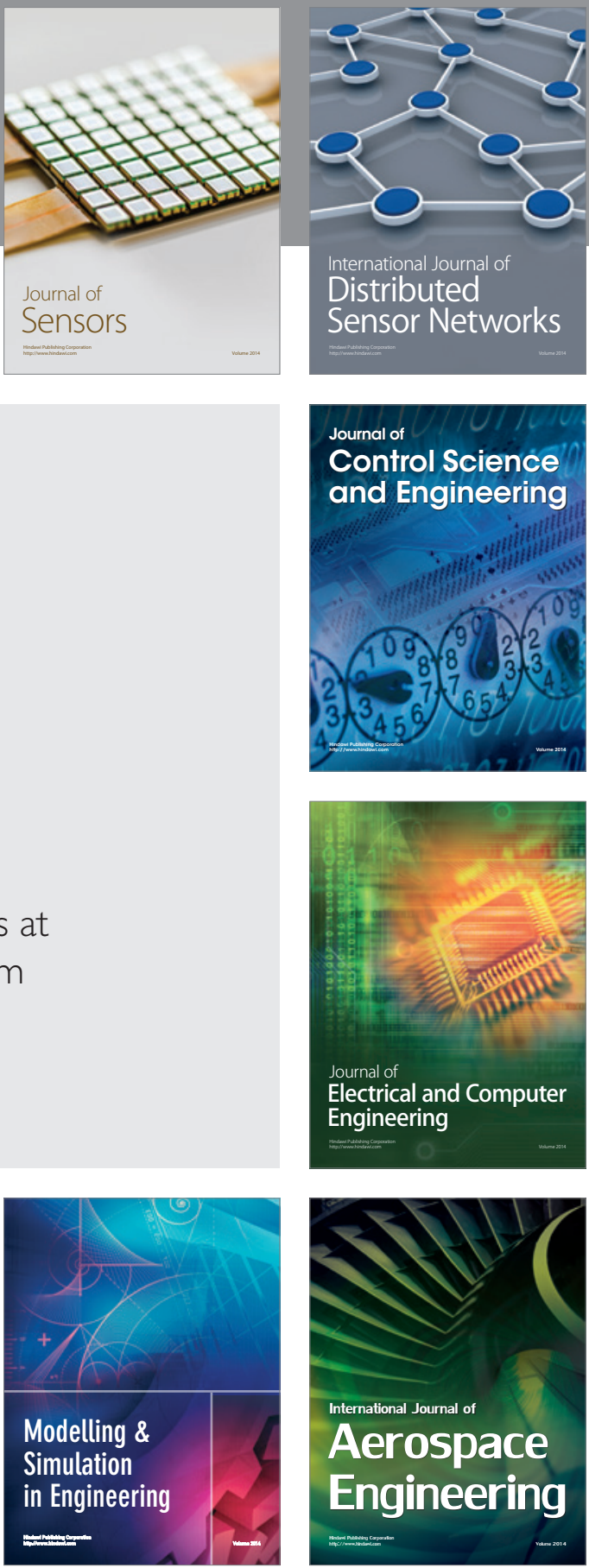

Journal of

Control Science

and Engineering
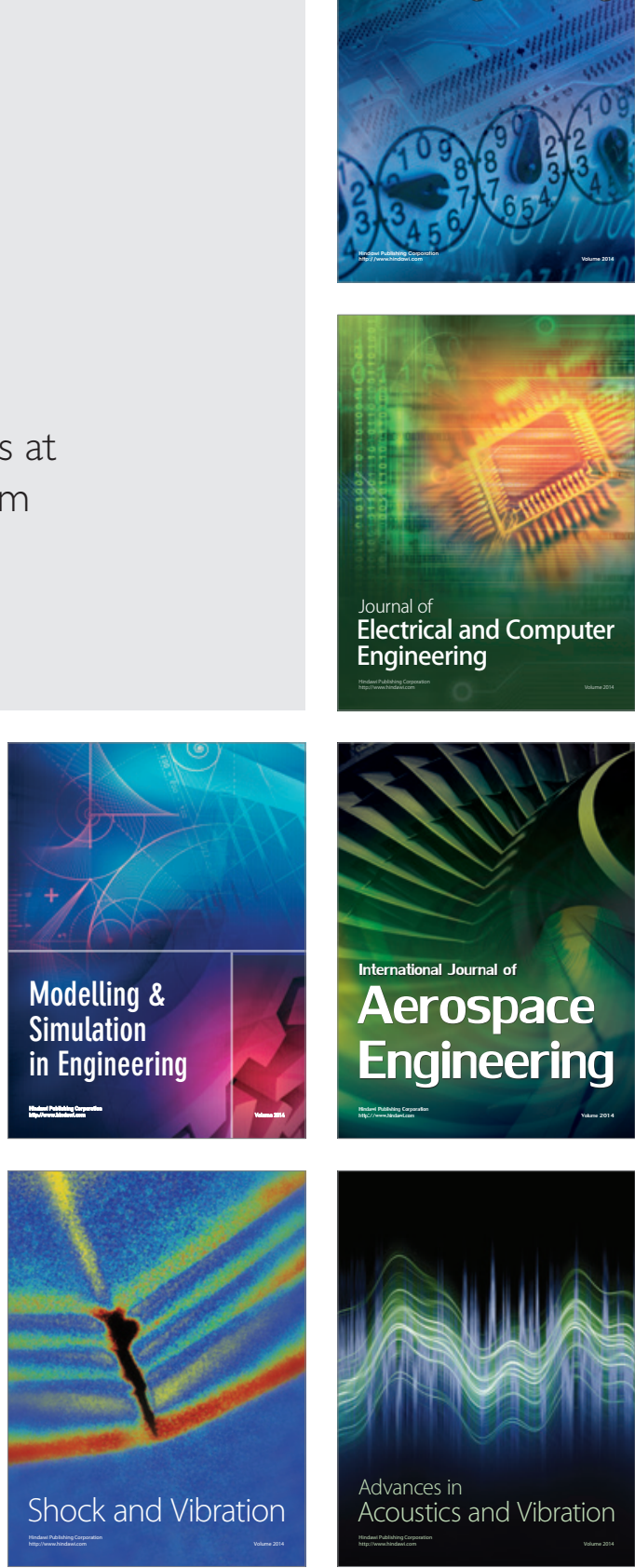